\title{
The Problem of Nephropathy Induced by Endocavitary Administration of lodine-Containing X-ray Contrast Agents
}

\author{
Iliya Mikhailovich Strekalov ${ }^{1}$, Leonid Petrovich Larionov², Mikhail Georgievich Zuev³, \\ Elena Yuryevna Zhuravleva ${ }^{4}$ \\ ${ }^{1}$ Central City Clinical Hospital No. 1, Ekaterinburg, Russia \\ ${ }^{2}$ Department of Pharmacology and Clinical Pharmacology, Faculty of Pharmacy, Ural State Medical University, Ekaterinburg, Russia \\ ${ }^{3}$ Oxide Systems Laboratory, Institute of Solid State Chemistry, Ural Branch of Russian Academy of Sciences, Ekaterinburg, Russia \\ ${ }^{4}$ Experimental Physics Department, Physico-Technical Institute, Ural Federal University Named After the First President of Russia B. N. \\ Yeltsin, Ekaterinburg, Russia
}

Email address:

istrekalov@mail.ru (I. M. Strekalov), leonid-larionov@mail.ru (L. P. Larionov), zuev@ihim.uran.ru (M. G. Zuev), zhu06@yandex.ru (E. Y. Zhuravleva)

\section{To cite this article:}

Iliya Mikhailovich Strekalov, Leonid Petrovich Larionov, Mikhail Georgievich Zuev, Elena Yuryevna Zhuravleva. The Problem of Nephropathy Induced by Endocavitary Administration of Iodine-Containing X-ray Contrast Agents. International Journal of Medical Imaging. Vol. 4, No. 3, 2016, pp. 17-22. doi: 10.11648/j.jimi.20160403.12

Received: June 16, 2016; Accepted: July 5, 2016; Published: July 18, 2016

\begin{abstract}
It is known that there is a high risk of progression of side effects in kidneys, so-called nephropathy induced with $\mathrm{X}$-ray contrast agents, at applying of water-soluble X-ray contrast agents in medical practice. New water-insoluble compounds of orthotantalates of rare-earth elements are offered as new X-ray contrast agents for endocavitary administration. The achieved results have shown the absence of general toxic effect on bodies of animals, high X-ray contrast properties in vitro and in vivo experiments. The investigations were performed on white nondescript mice, rats Wistar subtype and Chinchilla rabbits.
\end{abstract}

Keywords: X-ray Contract Agents, Nephropathy Induced with X-ray Contrast Agents, Orthotantalates of Rare-Earth Elements

\section{Introduction}

Side effects with the use of the X-ray contrast agents. The $\mathrm{X}$-ray contrast agents (XCA) used currently in medical practice are not absolutely safe. As all medical preparations, they can induce different hypersensitivity reactions, especially after parenteral administration. A concurrent reaction is any unwanted or unforeseen reaction on the administration of a drug requiring cure or alteration of the drug application conditions.

The sides effects (SE) of application of water-soluble XCA are observed for $5-8 \%$ of patients, and severe complications occur for $0.1 \%$ of patients. The fatal outcome ratio is from 1:40000 to 1:50000, and according to some data - to $1: 10000$ [1].

The development of kidney SE is due first of all to the chemical structure of compounds, the dose and concentration of solutions, the routes of their administration into the organism, the value of binding of XCA with seralbumin, the ability of XCA to be filtered in glomerules and to be secreted in tubules, as well as to the functional state of kidneys, which is subjected to different effects. Recently, the attention to kidney complications occurring as a result of application of XCA (contrast-induced nephropathy - CIN) increased considerably as the number of patients having initial kidney impairment grew. The risk factors include:

- kidney diseases in past history;

- genetic disposition to kidney diseases, pancreatic diabetes;

- hepatocellular failure;

- multiple myeloma;

- diffuse screloderma;

- administration of nephrotoxic preparations by patients (methmorphine, nonsteroidal antiinflammatory drugs, 
aminoglycosides etc.) [2].

In the USA, about 20 million people suffer from chronic kidney disease. This pathology is observed for $30 \%$ of all population over 60. Another large risk group of CIN development involves patients suffering from pancreatic diabetes. The number of such patients in the world exceeds 220 millions and, according to predictions, will be increased in 20 years' time by as many as $75 \%$. When nephropathy develops, the hospital stay time increases by $5-10$ days and medical expenses grow primarily due to the use of hemodialysis. CIN increases the mortality of patients (to $36 \%$ ) and 2-year survival rate is only $19 \%$. Against the background of chronic kidney disease and pancreatic diabetes, the risk of CIN increases by $33 \%$. Repeated X-ray contrast examination with iodine preparations leads to acute renal failure for $41 \%$ of patients, and in $10-15 \%$ cases the level of creatinin in serum is higher than $1.5 \mathrm{mg} / \mathrm{dl}(132.6$ $\mathrm{mcM} / \mathrm{l})$ [3].

Side effects with the use of iodine-containing XCA. Iodine-containing XCA (ICXCA) have a certain risk of nephrotoxicity for patients with normal renal function (7\%). If there are risk factors, the kidney SE rate is $20-30 \%$. [4].

According to the modern concepts, CIN develops with participation of several pathophysiological mechanisms:

- change in the rheological properties of blood;

- change in rental hemodynamics (tubular-glomerular feedback mechanism);

- development of regional hypothermia;

- activation of auto- and paracrine mediators (adenosine, endothelin, active forms of oxygen);

- direct cytotoxic action on renal tubule epithelium [2].

Studying the corresponding literature, we found little statistical information about SE associated with the use of water-soluble ICXCA in endocavitary administration routes during X-ray examination: cholangiography $(\mathrm{CG})$, oral duodenography, pyelography, cystography etc. Nevertheless, interesting comparative statistics of the SE development rate in X-ray examination with ionic XCA is reported by N. L. Shimanovskii, which is tabulated in work [2]:

Table 1. Comparative statistics of the SE development rate in X-ray examination with ionic XCA.

\begin{tabular}{llll}
\hline Examination & The quantity of patients & Side reaction rate (\%) & $\begin{array}{l}\text { The quantity of causa mortis (per the } \\
\text { quantity of examinations) }\end{array}$ \\
\hline Urography & 214033 & $10257(4.80)$ & $11(1: 20000)$ \\
Cholangiography & 33778 & $2678(8.00)$ & $2(1: 17000)$ \\
Cerebral angiography & 12771 & $263(2.06)$ & $1(1: 13000)$ \\
Angiocardiography & 7911 & $179(2.26)$ & $2(1: 14000)$ \\
Aortography & 24885 & $665(2.67)$ & $1(1: 25000)$ \\
Other types of angiography & 2815 & $101(3.58)$ & 0 \\
All types of angiography & 48382 & $1208(2.5)$ & $4(1: 12000)$ \\
\hline
\end{tabular}

Analysis of these data revealed some regular features:

1). the largest number of SE induced by CG as compared with the other types of X-ray contrast examination (almost twice as often as after intravenous urography and thrice as often as after angiography);

2). the causa mortis rate after X-ray contrast studies of bile passages exceeds that induced by intravenous urography and is slightly smaller than after angiography.

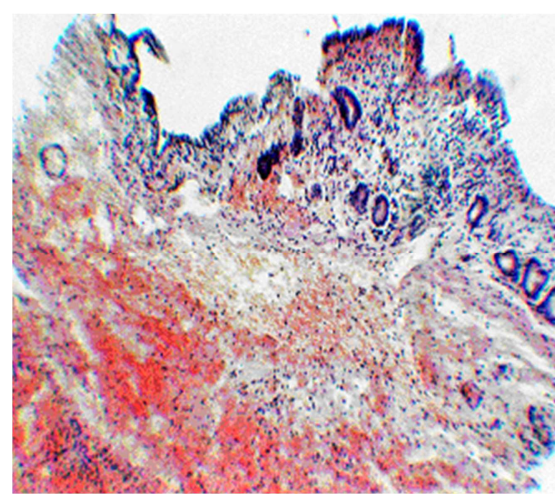

Fig. 1. A gall bladder of a dog upon administration of 30\% urografin and 1 h exposure: extensive erosions and ulcers of mucous tunic, hemorrhages and apparent leucocytic infiltration of lamina propria. Hematoxylin and eosin stain. $x 100$ magnification.
The main releasing mechanism of CIN in endocavitary administration routes is the ability of water-soluble ICXCA to be adsorbed into blood through epithelial membranes. This concerns first of all the gastrointestinal tract, as well as abdominal, pleural and intraacticular cavities, urinary tracts and other natural abdominal cavernous organs. XCA contact with the contents of hollow organs and undergo chemical changes. In this form, the water-soluble fractions of XCA penetrate owing to the lymphatic bed into the lymphatic system and then into the blood channels and further are egested mainly through kidneys. Here, the same mechanisms of toxic action are involved as in direct intravascular administration of XCA [5].

It is important that many patients (probably the majority) that need X-ray examination of bile passages initially have biliary hypertension as the main pathophysiological mechanism of obstructive jaundice. Intraductal hypertension provides conditions for reabsorption of bile components into the blood stream. Besides, water-soluble ICXCA exert a local irritant and cytotoxic effect on the mucous tunics of bile passages, which we observed repeatedly in experiments and described earlier (Fig. 1) [6]. Injury of mucous tunics promotes even more intensive penetration of duct contents into the lobular system of liver and further into blood thus 
resulting in even greater "toxic load" on kidneys.

When hepatic disorders, especially detoxicative hepatic dysfunction, occur, kidneys partly make up for metabolisation of many xenobiotics. Under these conditions, nephrotoxicity of XCA rises sharply and the probability of CIN development increases considerably. Thus, patients liable to X-ray endoscopic treatment of bile passages are another risk group of CIN development.

Search for safest X-ray contrast compositions. In order to reduce complications in the application of XCA, it is necessary to search for safer X-ray contrast compositions. The main research trend has to do with improvement of the ICXCA group [2, 5].

Theoretically, the safest preparation with respect to toxic properties of XCA for endocavitary administration should be water-insoluble. Such preparation cannot get into the blood stream and, consequently, no general toxic complications can be developed.

The widely used barium suspension, being a waterinsoluble preparation, is devoid of toxic properties inherent in ICXCA. However it has some drawbacks. Numerous cases have been described when toxic and infectious inflammatory reactions developed because barium suspension accidentally got into the respiratory tracks, bile ducts or the abdominal cavity. Barium suspension may stay for a long time in sinus tracts, diverticula or vermiform appendix resulting in suppurative complications. When there are marked stenoses, barium suspension creates danger of development of gross obstruction. Consequently, barium suspension is not indicated in such cases [5].

There are works devoted to the application of iodine-free preparations as XCA. The gadolinium-based preparation for magnetic resonance imaging - gadoliniumdiethylenetriaminopentaacetate (Gd-DTPA) - was used by T. Velmas and T. Markkola for endoscopic retrograde pancreatic cholangiography, fistulography, cystography, urethrocystography and antegrade pyelography. Most of the authors are of the opinion that the X-ray images produced with Gd-DTPA are inferior in quality (first of all in contrast) to those obtained with the use of iodine-containing XCA. At the same time, SE of general toxical character were registered during such examinations, although they took place more rarely than for the "traditional" contrast agents [7].

In terms of X-ray contrast properties, of much interest are the compounds of rare-earth elements $(\mathrm{Y}, \mathrm{La}, \mathrm{Gd})$ and tantalum [8 - 13].

\section{Materials and Methods}

Orthotantalates of lanthanum (LOT), yttrium (YOT) and gadolinium (GOT) have been synthesized by the solid-state method from $\mathrm{La}_{2} \mathrm{O}_{3}, \mathrm{Y}_{2} \mathrm{O}_{3}, \mathrm{Gd}_{2} \mathrm{O}_{3}$ and $\mathrm{Ta}_{2} \mathrm{O}_{5}$ oxides. The gel form was obtained in accordance with RU patent No. 2261114.

Acute toxicity, local irritant and sensibilizing action, as well as the effect of the preparations on urinary excretion in long-term (180 days) experiment have been studied. Powder-like XCA with particle diameter not more than 8 $\mathrm{Mm}$ were used as $50 \%$ supernatant fluid, $25 \%$ water suspension and $25 \%$ gel suspension. The investigations were performed on white nondescript mice, rats Wistar subtype and Chinchilla rabbits. The general condition of the animals, their appetite, body weight, behavior and the state of the main systems of the organism were observed throughout the experiment. A histological study of the animals' tissues was carried out in the course of the experiment.

Besides, the ability of XCA to be penetrated into blood and egested by the urinary system of rats was examined. For this purpose, X-ray studies of urine samples of rats from the experimental and the control groups were performed during and on completion of long-term experiment.

The X-ray contrast properties of the compounds were examined in vitro and in vivo with the use of radiography. The preparations were made with $0.25-30 \%$ concentrations. The results of the X-ray studies were estimated visually and with the aid of densitometry.

\section{Results and Discussion}

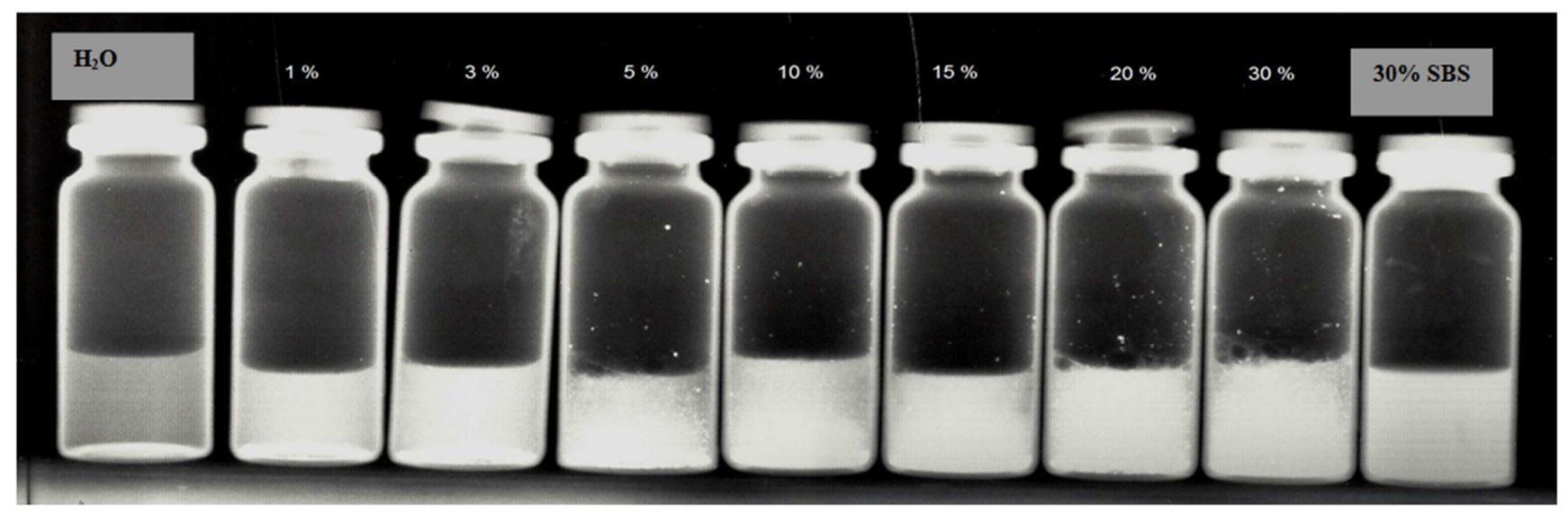

Fig. 2. Comparative characteristic of X-ray contrast properties of gel suspension of GOT with different concentrations and the standard barium suspension. 


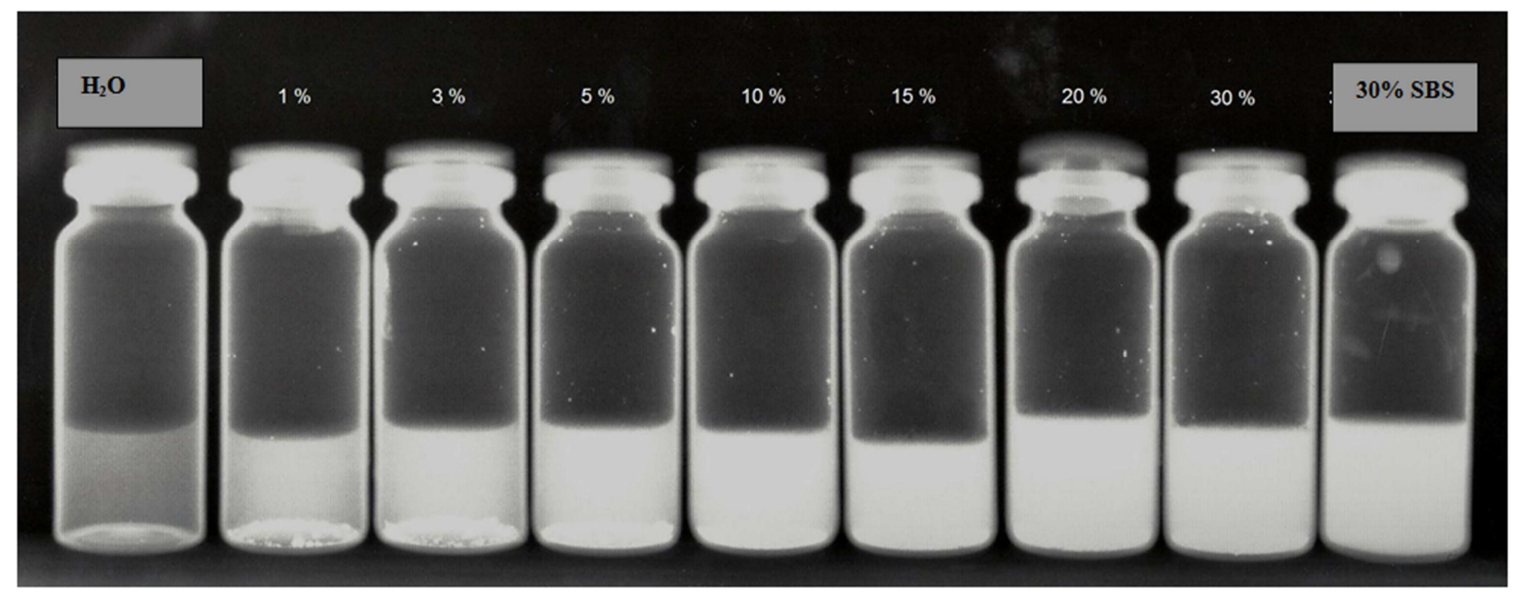

Fig. 3. Comparative characteristic of X-ray contrast properties of gel suspension of GOT with different concentrations and $30 \%$ urografin.

Acute and chronic toxicity of XCA was estimated and no death of animals was registered. Analysis of the obtained results showed that the XCA have no irritant or sensibilizing action both at single and long-term exposure. Autopsy and macro- and microscopic examinations revealed no morphlogical changes in the organs including kidneys, ureters and urinary bladder. Examining X-ray contrast of animals' urine after long-term experiment we did not obtain any reliable data that in the control group the contrast was better.

For comparative estimation of the X-ray contrast properties of LOT, YOT and GOT we used $1 ; 3 ; 5 ; 10 ; 15 ; 20$ and $30 \%$ concentrations of each XCA. For each series of Xray films we took the same concentration of each XCA. No reliable differences in the degree of shadowing of the X-ray films were found by visual evaluation. However, reliable advantage of X-ray contrast for the samples of the gel suspension of GOT was determined by densitometry.

The in vitro examination of the X-ray contrast properties of XCA was continued by comparing them with the properties of the standard barium suspension (SBS) and $30 \%$ urografin. $1 ; 3 ; 5 ; 10 ; 15 ; 20$ and $30 \%$ concentrations of gel-

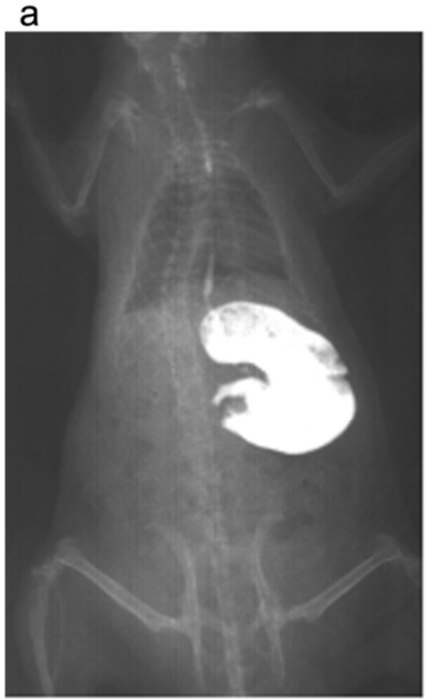

b
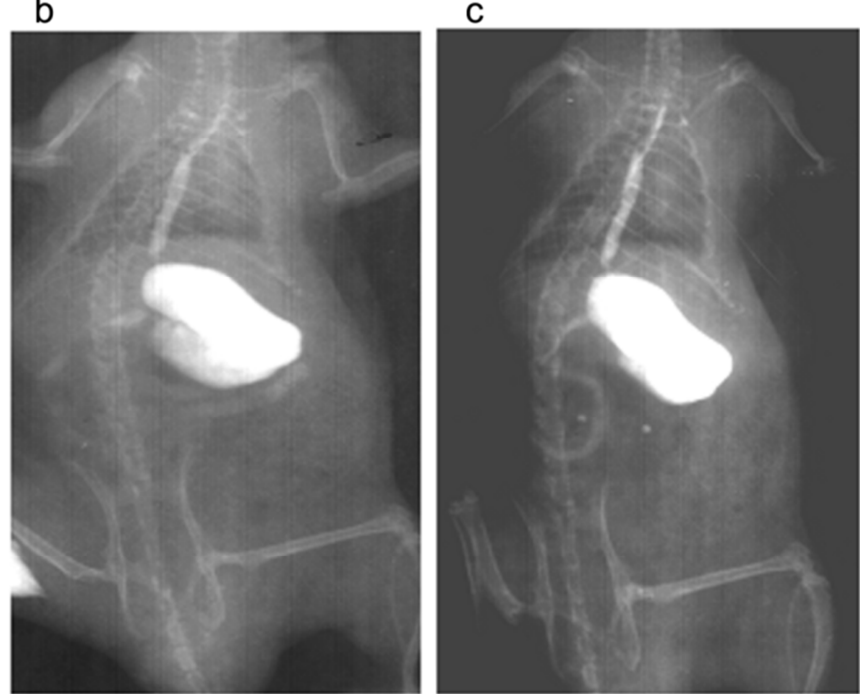

Fig. 4. Contrast study of esophagus, stomach and duodenum of a rat: $30 \%$ gel suspension of GOT (a), standard barium suspension (b) and $30 \%$ urografin (c). 
The X-ray studies were performed directly after the administration of preparations into the stomach of rats and 6, 12, 24 and $48 \mathrm{~h}$ later. Experiments in vivo in contrasting the gastrointestinal tract were performed on two groups of rats of both sexes, line subtype Vistar (10 animals per group). By using a metallic probe was injected $5 \mathrm{ml}$ a 20,25 and $30 \%$ gel suspension of OTL. The results were evaluated visually. When 20, 25 and $30 \%$ gel suspensions of LOT, YOT and
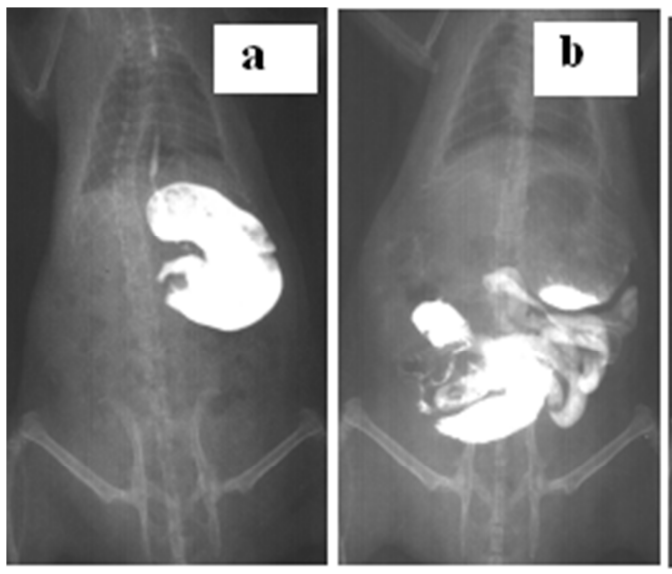

GOT were used, sharp contours of the esophagus, stomach and small intestine were obtained on the X-ray films. The Xray image obtained with the use of $30 \%$ gel suspensions of the above preparations corresponds to that produced with the aid of $30 \%$ urografin and is superior to that acquired with the help of SBS in intensity, contrast and definition (Fig. 4). Complete evacuation of XCA was registered in 24-48 h depending on the concentration (Fig. 5).

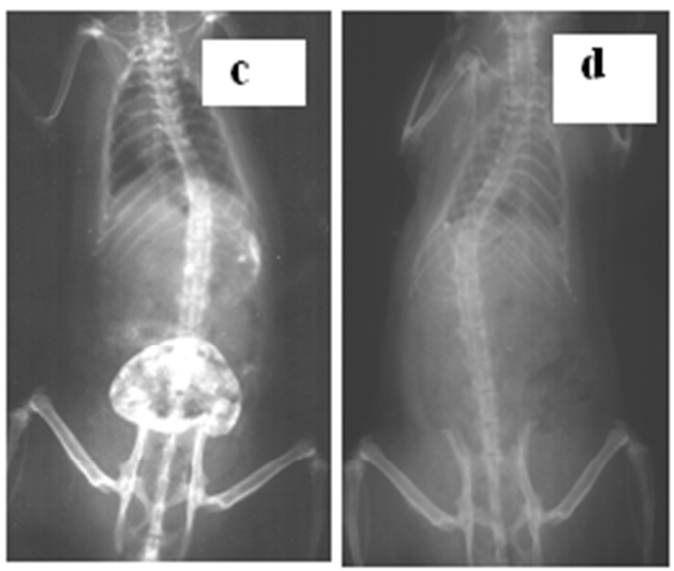

Fig. 5. The XRD patterns of rat after contrasting of the gastrointestinal tract a 30\% OTL: directly after administration (a), 12 (b), 24 (c) and 48 hours (d) after contrasting.

Thus, the studies performed revealed that the gel-based $\mathrm{XCA}$ are not toxic with respect to the organism of animals and do not exert any side effects typical of iodine-containing XCA including those affecting the urinary system. They have a pronounced ability to absorb X-rays and give sharp images in X-ray studies of hollow organs of animals. The image contrast is not inferior to that provided by SBS and urografin. No additional medical equipment is required to perform diagnostic examination with the use of the considered XCA.

The optimal concentration of LOT, YOT and GOT for Xray examination of rats' stomach is $25 \%$ and $30 \%$.

This shows that the gel-based LOT, YOT and GOT hold promise as X-ray contrast agents for contrast studies of hollow organs first of all in endoscopic retrograde pancreatic cholangiography, intraoperative, transfistula cholangiography, retrograde and antegrade pyelography and cystography.

\section{Conclusions}

a. The synthesized water-insoluble XCA do not exert general toxic action on the organism of animals, do not affect the kidney structure and function both during single and multiple application.

b. The XCA have no local irritant or cytotoxic action when contacting with mucous tunics.

c. In the group of XCA, GOT possess the highest X-ray contrast properties.

d. The XCA based on REE orthotantalates show promise for X-ray contrast diagnostic examination and high-tech $\mathrm{X}$-ray endoscopic interventions in cavitary structures of man. This is due to the fact that these XCA have no general toxic action on the organism and, first of all, on the anatomico-functional state of kidneys. Moreover, the new XCA exhibit high X-ray contrast properties, have low cost and do not require additional X-ray and endoscopic equipment.

\section{References}

[1] Rosenstrauch L. S. Prevention of complications in X-ray studies with the use of contrast agents / L. S. Rosenstrauch // Vestnik rentgenologii i radiologii. 1978. No. 9, pp. $31-35$. [in Russian].

[2] Shimanovskii N. L. Contrast agents. Efficient application guide. Moscow: GEOTAR-Media. 2009. 463 p. [in Russian].

[3] John A. K. Renal insufficiency: gadopentetate dimeglumine as a radiographic contrast agent during peripheral vascular interventional procedures / A. K. John, C. G. Stuart, C. W. Arthur // Radiology. 1996. Vol. 198, pp. $579-581$.

[4] Gomes A. S. Acute renal dysfunction in high-risk patients after angiography: comparison of ionic contrast media / A. S. Gomes, I. F. Lois, I. D. Baxer // Radiology. 1989. Vol. 121, No. 14 , pp. $410-415$.

[5] Sergeyev P. V., Sviridov N. K., Shimanovskii N. L. X-ray contrast agents. Moscow: Meditsina. 1993. 307 p. [in Russian].

[6] Strekalov I. M. A new X-ray contrast agent - lanthanum orthotantalate and possibilities of its application in abdominal surgery (experimental study): candidate of science (medicine) thesis / I. M. Strekalov / Ekaterinburg. 2007, pp. 84 - 87. [in Russian]. 
[7] Velmas T. / Gd-DTPA as an alternative contrast agent in conventional and interventional and radiology / T. Velmas $\mathrm{T}$. Markkola // Acta Radiol. 1998. Vol. 39, pp. 223 - 226.

[8] Zuev M. G., Larionov L. P. Tantalum X-ray contrast agents (Solid-State Chemistry for Medicine Series). Ekaterinburg: Ural. Otd., Ross. Akad. Nauk, 2002. ISBN 5-7691-1282-4. [in Russian]. 155 p.

[9] New Materials for Medicine / Collective of authors, M. G. Zuev and L. P. Larionov, Eds., Eekaterinburg: Ural. Otd., Ross. Akad. Nauk, 2006. ISBN 5-7691-1718-4. [in Russian]. $166 \mathrm{p}$.

[10] Zhuravleva E. Yu. Tantalum X-ray Contrast Media (Solid-
State Chemistry for Medicine Series), by M. G. Zuev and L. P. Larionov, Eekaterinburg: Ural. Otd., Ross. Akad. Nauk, 2002 // Inorganic Materials, Vol. 40, No. 6, 2004, pp. $671-672$.

[11] Zhuravleva E. Yu. New Materials for Medicine, M. G. Zuev and L. P. Larionov, Eds., Eekaterinburg: Ural. Otd., Ross. Akad. Nauk, 2006 // Inorganic Materials, 2007, Vol. 43, No. 9, p. 1034.

[12] Zuev M. G. and Larionov L. P. "Tantalic Radio-Opaque Drugs. Preclinical Studies" Monography. Lambert Academic Publishing, 2012. 250 p. [in Russian].

[13] Strekalov I. M., Larionov L. P., Zuyev M. G. // Vestnik rentgenologii i radiologii. 2007 NO 1, pp. $45-54$. 